\section{Accurate measurements of liver stiffness using shear wave elastography in children and young adults and the role of the stability index}

\author{
Eun Kyoung Hong ${ }^{1}$, Young Hun Choi ${ }^{1,2}$, Jung-Eun Cheon ${ }^{1,2,3}$, Woo Sun Kim ${ }^{1,2,3}$, \\ In-One Kim ${ }^{1,2,3}$, Sun Young Kang ${ }^{4}$ \\ ${ }^{1}$ Department of Radiology, Seoul National University Hospital, Seoul; ${ }^{2}$ Department of \\ Radiology, Seoul National University College of Medicine, Seoul; ${ }^{3}$ Institute of Radiation \\ Medicine, Seoul National University Medical Research Center, Seoul; ${ }^{4}$ Department of Clinical \\ Ultrasound, DongSeo Medicare Co., Ltd., Seongnam, Korea
}

Purpose: The purpose of this study was to evaluate the usefulness of the stability index (SI) in liver stiffness measurements using shear wave elastography (SWE) in children.

Methods: A total of 29 children and young adults (mean age, 16.1 years; range, 8 to 28 years; 11 boys and 18 girls) who underwent liver stiffness measurements using SWE under freebreathing and breath-holding conditions were included in our study. Ten SWE measurements were acquired in each of four groups: free-breathing and breath-holding, and with and without using the SI. The failure rate of acquisition of SI values over $90 \%$ was calculated in each group. To evaluate variability in the SWE measurements, the standard deviation, coefficient of variation, and percentage of unreliable measurements were compared. Intraobserver agreement and the optimal minimal number of measurements were calculated using intraclass correlation coefficients.

Results: A failure to acquire SI values over $90 \%$ was observed in $17 \%$ of the scans in the freebreathing group and in $7 \%$ of the scans in the breath-holding group. In both groups, utilizing the SI led to a significantly lower standard deviation and coefficient of variation. When using the $\mathrm{SI}$, the percentage of unreliable measurements decreased from $16.7 \%$ to $8.3 \%$ in the free-breathing group and $14.8 \%$ to $0 \%$ in the breath-holding group. With the use of the $\mathrm{SI}$, intraobserver agreement increased and the optimal minimal number of repeated measurements decreased in both the free-breathing and breath-holding groups.

Conclusion: Utilization of the SI in the measurement of liver SWE in children reduced measurement variability and increased reliability in both free-breathing and breath-holding conditions.

Keywords: Elastography; Shear wave elastography; Stability index; Liver; Children

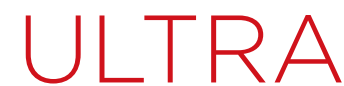

SONO

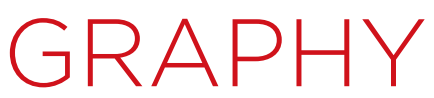

ORIGINAL ARTICLE

https://doi.org/10.14366/usg. 17025 pISSN: 2288-5919 - eISSN: 2288-5943

Ultrasonography 2018;37:226-232

Received: March 9, 2017

Revised: August 1, 2017

Accepted: August 19, 2017

Correspondence to:

Young Hun Choi, MD, Department of Radiology, Seoul National University Hospital, 101 Daehak-ro, Jongno-gu, Seoul 03080, Korea

Tel. +82-2-2072-3608

Fax. +82-2-747-5781

E-mail: iater@snu.ac.kr

This is an Open Access article distributed under the terms of the Creative Commons Attribution NonCommercial License (http://creativecommons.org/ licenses/by-nc/3.0/) which permits unrestricted noncommercial use, distribution, and reproduction in any medium, provided the original work is properly cited.

Copyright (C) 2018 Korean Society of Ultrasound in Medicine (KSUM)

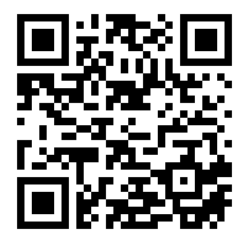

How to cite this article:

Hong EK, Choi YH, Cheon JE, Kim WS, Kim IO, Kang SY. Accurate measurements of liver stiffness using shear wave elastography in children and young adults and the role of the stability index. Ultrasonography. 2018 Jul;37(3):226-232. 


\section{Introduction}

Fibrosis or cirrhosis of the liver is a manifestation of end-stage liver disease, which is a common outcome of various chronic liver diseases in children. As hepatic fibrosis can be lethal and can induce various complications in children, the precise evaluation of liver fibrosis is very important in patient management and prognosis $[1,2]$. Even though liver biopsy has been considered the standard for evaluating the degree of hepatic fibrosis, there are several restrictions on the use of liver biopsy in children due to its invasiveness, potential complications, sampling variations, and related interobserver and intraobserver variability [3]. Therefore, liver biopsy is generally conducted in children in limited circumstances, and there is a constant need to search for a noninvasive method to measure liver stiffness such as ultrasound (US) or magnetic resonance elastography techniques $[4,5]$. Among such techniques, US-based elastography has been very promising in children because of its noninvasiveness, easy accessibility, and real-time nature $[5,6]$. US-based elastography techniques have made it possible to acquire quantitative measurements of liver fibrosis [7] within a short time period in routine sonographic examinations, even during free breathing [8]. Several real-time US-based elastography techniques have been introduced, and studies have demonstrated a high correlation between US-based elastography and the severity of liver fibrosis in children $[2,9]$.

Shear wave elastography (SWE; Aixplorer, SuperSonic Imagine SA, Aix-en-Provence, France), one of several real-time US-based elastography techniques, provides quantitative tissue elasticity maps. With SWE, shear waves are generated from acoustic pulses forced at five different tissue depth levels $[8,10]$ and the shear wave velocity is estimated by ultrafast Doppler-like acquisition of 5,000 frames per second. Then, the shear wave speed is converted to tissue stiffness as kilopascals using the formula $E=3 \rho v^{2}$, where $E$ represents the elasticity of the tissue, $\rho$ represents the density of the tissue, and $v$ represents shear wave velocity. Although several studies have been conducted regarding the reproducibility and reliability of SWE in measuring liver fibrosis in adults $[11,12]$, not much is known about the reproducibility of SWE in pediatric patients. Furthermore, most studies have analyzed data obtained using the breath-holding technique during the measurement of liver stiffness with SWE. Using breath-holding techniques can potentially minimize artifacts and disturbances in shear wave velocity acquisition caused by movement during breathing [13]. Since it can be difficult to achieve adequate breath-holding during the acquisition of US-based elastography in young children, it is necessary to compare liver stiffness measurements during breath-holding and free-breathing and to investigate methods that can assist in the acquisition of adequate measurements of hepatic fibrosis in children. The stability index (SI) is a new technique that provides temporal stability when placing a region of interest (ROI) box during SWE examinations, in order to ensure a high-quality assessment of liver stiffness by analyzing the temporal and spatial reproducibility of the last three to five images to create a SI value. An SI value over $90 \%$ is considered a good result, and indicates adequate acquisition of data within the ROI. We hypothesized that using this new technique would improve the reproducibility of liver SWE measurements in children. To our knowledge, no study has evaluated the clinical application of the SI in measuring liver stiffness using SWE.

Therefore, the purpose of our study was to evaluate the usefulness of the SI for decreasing the variability of liver stiffness measurements and the number of measurements required for adequate data acquisition free-breathing and breath-holding conditions in children.

\section{Materials and Methods}

\section{Study Population}

This was a retrospective study. Our Institutional Review Board approved the study, and the requirement for informed consent was waived (IRB No. 1702-071-831). From August to December 2016, one radiologist (Y.H.C., with 13 years of experience in US imaging) performed liver ultrasonography including SWE in 52 consecutive children and young adults. Among them, a total of 29 children and young adults (mean age, 16.1 years; range, 8 to 28 years; 11 boys and 18 girls) who underwent liver stiffness measurements using SWE in both free-breathing and breath-holding conditions were included in this study. The indications for liver ultrasonography in the children included in the study were as follows: fatty liver $(n=4)$, cardiac cirrhosis $(n=7)$, Wilson disease $(n=4)$, chronic liver disease $(n=3)$, glycogen storage disease $(n=4)$, post-bone marrow transplant surveillance $(n=1)$, fever work-up $(n=1)$, and malignancy follow-up $(n=5)$.

\section{SWE Technique}

One radiologist (Y.H.C., with 13 years of experience in US imaging) performed the SWE examination with a single US scanner (Aixplorer, SuperSonic Imagine SA) using the same convex broadband probe (XC6-1), following the protocol presented below. All patients were told to fast for at least 4 hours before the examination and were examined in a supine position with upward extension of the right arm over their heads. The probe was gently placed over the right intercostal space and an approximately $2.0 \times 2.5-\mathrm{cm}$ SWE box was placed in the right lobe of the liver, $1.5-2 \mathrm{~cm}$ away from the Gleason capsule to avoid reverberation artifacts and pulsation from adjacent vascular structures. Afterwards, two SWE video clips of 
$10-15$ seconds (7-10 frames per second) were obtained for each patient in the same order, as follows. The first SWE video clip was measured when the patients lay on the examination table in the supine position and were given instructions to hold their breath in the mid-expiratory phase for as long as they could for the acquisition of the SWE video in the breath-holding study. After a sufficient time interval for patients to catch their breath, the patients were not given any instructions that might exaggerate respiration for the second acquisition in the free-breathing study, and SWE video was obtained for an equal time duration to that of the breath-holding study.

\section{Liver Stiffness Measurements}

The SWE video clips were retrospectively reviewed by another radiologist (E.K.H., with 3 years of experience in US imaging), who was blinded to the patient's history. A $15-\mathrm{mm}$ circular ROI was placed carefully in the area where the color-coding was as homogeneous as possible, in order to avoid the inclusion of any artifacts related to motion or pulsation. Mean, minimum, and maximum elasticity values in kilopascals and standard deviation within the ROI, as well as information about the depth and diameter of the ROI and the SI value were automatically displayed on the monitor by placing a circular ROI within the box on the SWE image (Fig. 1). The manufacturer's recommendations for obtaining reliable

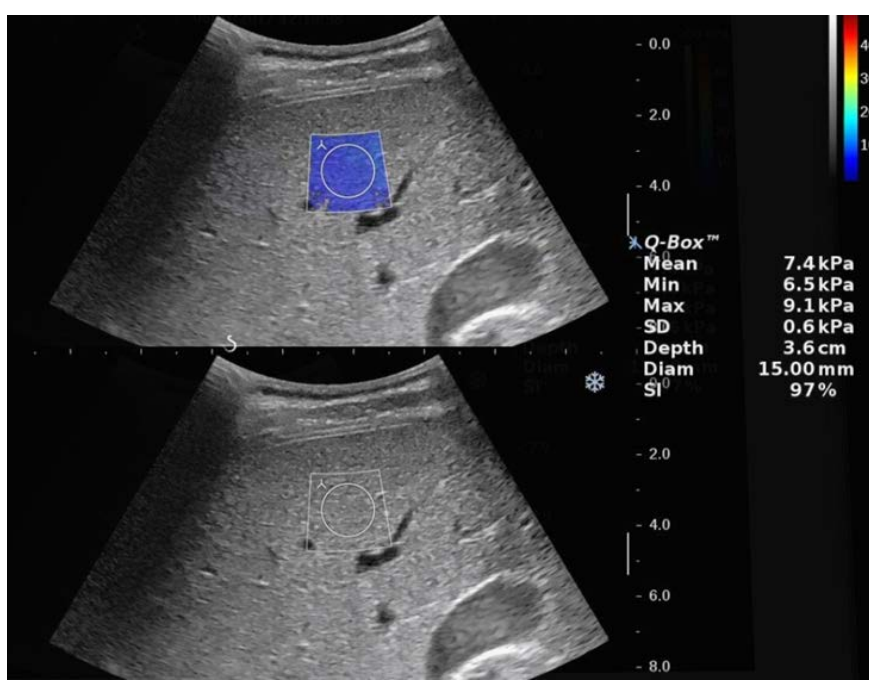

Fig. 1. Elastography of a 14-year-old boy who underwent abdominal ultrasonography for evaluation of glycogen storage disease. The B-mode image and shear wave elastography (SWE) elastographic images were placed up and down. A SWE box $(2.0 \times 2.5$ $\mathrm{cm}$ sized) was placed in the right anterior segment of the liver, avoiding vascular structures. After the entire area of SWE box was color-coded, $15-\mathrm{mm}^{2}$ circular region of interest was placed carefully in the area where the color coding was as homogeneous as possible not to include any artifacts related to motion or pulsation. data are to perform three valid independent 2-dimensional SWE measurements and to use the mean value of these measurements [14]. In this study, considering the immaturity of pediatric patients, a total of 10 measurements of mean liver elasticity and standard deviation values for each free-breathing and breath-holding group were acquired, regardless of the SI value. Another 10 measurements were performed for each free-breathing and breath-holding group using the $\mathrm{SI}$, where only ROls with an $\mathrm{SI}$ value of over $90 \%$ were taken into consideration for evaluation. Each measurement was separated by at least five frames from each other. Finally, the following four groups of 10 measurements were formed in order to evaluate the difference in values acquired with or without using the SI according to the respiratory state: free-breathing without the SI (FBeye), free-breathing with the SI (FBSI), breath-holding without the SI (BHeye), and breath-holding with the SI (BHSI).

\section{Data Analysis}

The rate of failure of acquisition of SI values over $90 \%$ was calculated for each free-breathing and breath-holding study. The SWE measurement was considered to have failed when it was impossible to acquire 10 ROls with SI values over $90 \%$ throughout the whole series of the SWE cine clip. The mean SWE values of each dataset were compared using the paired t-test, to see if there was any difference between free-breathing and breath-holding or depending on the usage of the SI.

Variability in SWE measurements was evaluated using the following three methods. First, standard deviation values were compared with and without the SI for each free-breathing and breath-holding group. Second, the coefficient of variance was calculated to characterize the dispersion of the measurements. The coefficient of variance was defined as the ratio of the standard deviation to the mean, which represents a standardized measure of variability related to the mean [15]. Third, the percentages of unreliable measurements with or without $\mathrm{SI}$ were calculated. When the interquartile range (IQR) divided by the median liver stiffness value from the 10 measurements in each dataset was greater than $30 \%$ (IQR/median liver stiffness $>30 \%$ ), the SWE measurement was assumed to be unreliable because the variability of the measured stiffness values was too great [11].

The intraobserver agreement was evaluated by comparing the results of the first five and the last five measurements in each group using intraclass correlation coefficients (ICCs) [16]. Intraobserver reproducibility was considered as poor (ICC, 0.00 to 0.20$)$, fair to good (ICC, 0.40 to 0.75$)$, or excellent (ICC, $>0.75$ ) [17].

Finally, to evaluate the minimal optimal number of measurements, subsets of the first two to nine serial measurements were selected. The median liver stiffness values of these eight subsets were 
compared with the reference liver stiffness (median liver stiffness of the 10 measurements in each dataset) by using ICCs. The minimal number of SWE acquisitions for which there was no significant difference between the ICCs and the $95 \%$ confidence intervals ( $\mathrm{Cls}$ ) in multiple comparisons was considered to be the optimal number of acquisitions.

All statistical analyses were performed with MedCalc (ver. 12.1.0 for Microsoft Windows 2000/XP/Vista/7, MedCalc Software, Mariakerke, Belgium) or R (ver. 3.3.1, R Foundation for Statistical Computing, Vienna, Austria). Results with a P-value of less than 0.05 were considered statistically significant.

\section{Results}

Failure of acquisition of SI values over $90 \%$ was observed in five

Table 1. Shear wave elastography values measured in four groups

\begin{tabular}{lcc}
\hline & Mean \pm SD & No. of patients \\
\hline Free-breathing with SI & $6.38 \pm 4.39$ & 24 \\
Free-breathing without SI & $6.31 \pm 3.98$ & 29 \\
Breath-holding with SI & $6.72 \pm 4.70$ & 27 \\
Breath-holding without SI & $6.47 \pm 4.09$ & 29 \\
\hline
\end{tabular}

SD, standard deviation; SI, stability index. of 29 patients (17\%) in the free-breathing group and in two of 29 patients $(7 \%)$ in the breath-holding group.

Comparison of the mean elasticity value revealed no significant difference between free-breathing and breath-holding $(6.31 \pm 3.98$ $\mathrm{kPa}$ vs. $6.47 \pm 4.09 \mathrm{kPa}, \mathrm{P}=0.354, \mathrm{n}=29$ ). There was no significant difference in the mean elasticity value between the FBeye and FBSI groups (6.31 $\pm 3.98 \mathrm{kPa}$ vs. $6.38 \pm 4.39 \mathrm{kPa}, \mathrm{P}=0.694, \mathrm{n}=24)$ or between the BHeye and BHSI groups $(6.47 \pm 4.09 \mathrm{kPa}$ vs. $6.72 \pm 4.70$ $\mathrm{kPa}, \mathrm{P}=0.101, \mathrm{n}=27$ ) (Table 1).

Table 2 summarizes the differences in the variability of SWE measurements between the groups that utilized the SI and the groups that did not. In the comparison of the acquired standard deviation values with and without utilization of the $S I$ in both the free-breathing and breath-holding groups, the groups that utilized SI showed significantly lower standard deviation values than those that did not $(\mathrm{P}<0.01)$.

In both the free-breathing and breath-holding groups, the coefficient of variation was significantly lower when using the $\mathrm{SI}$ than when not using the SI (free-breathing group, $0.055 \pm 0.230$ vs. $0.182 \pm 0.022, P<0.01$; breath-holding group, $0.037 \pm 0.223$ vs. $0.198 \pm 0.125, P<0.01)$.

Four of the 24 SWE measurements (16.7\%) were considered unreliable in the free-breathing group. When the SI was used, the number of patients with unreliable results decreased to two of the

Table 2. Indexes of variability in shear wave elastography measurements according to usage of the stability index

\begin{tabular}{lcccccc}
\hline & FBSI $(\mathrm{n}=24)$ & FBeye $(\mathrm{n}=24)$ & P-value & BHSI $(\mathrm{n}=27)$ & BHeye $(\mathrm{n}=27)$ & P-value \\
\hline Mean \pm SD & $1.03 \pm 1.69$ & $2.31 \pm 1.97$ & $<0.001$ & $0.65 \pm 0.59$ & $1.76 \pm 1.84$ & 0.001 \\
Coefficient of variation & $0.06 \pm 0.23$ & $0.19 \pm 0.02$ & $<0.001$ & $0.04 \pm 0.22$ & $0.20 \pm 0.13$ & $<0.001$ \\
Unreliable measurement, $\mathrm{n}(\%)$ & $2 / 24(8.3)$ & $4 / 24(16.7)$ & 0.383 & $0 / 27(0.0)$ & $4 / 27(14.8)$ & 0.038
\end{tabular}

FBSI, during free-breathing, using the stability index; FBeye, during free-breathing, without using the stability index; BHSI, during breath-holding, using the stability index; BHeye, during breath-holding, without using the stability index.

Table 3. ICCs and $95 \%$ Cls between eight subsets of two to nine consecutive measurements and the 10 reference measurements

\begin{tabular}{|c|c|c|c|c|c|c|c|c|}
\hline \multirow{2}{*}{ Repetition } & \multicolumn{2}{|c|}{ FBSI } & \multicolumn{2}{|c|}{ FBeye } & \multicolumn{2}{|c|}{ BHSI } & \multicolumn{2}{|c|}{ BHeye } \\
\hline & ICC & $95 \% \mathrm{Cl}$ & ICC & $95 \% \mathrm{Cl}$ & ICC & $95 \% \mathrm{Cl}$ & ICC & $95 \% \mathrm{Cl}$ \\
\hline 2 & 0.92 & $0.81-0.96$ & 0.88 & $0.76-0.94$ & 0.99 & $0.99-0.99$ & 0.91 & $0.83-0.96$ \\
\hline 3 & 0.93 & $0.89-0.98$ & 0.88 & $0.76-0.94$ & 0.99 & $0.99-0.99$ & 0.93 & $0.85-0.96$ \\
\hline 4 & 0.95 & $0.88-0.99$ & 0.89 & $0.77-0.95$ & 0.99 & $0.99-0.99$ & 0.92 & $0.84-0.96$ \\
\hline 5 & 0.96 & $0.91-0.98$ & 0.90 & $0.80-0.95$ & 0.99 & $0.99-0.99$ & 0.93 & $0.86-0.97$ \\
\hline 6 & 0.96 & $0.92-0.98$ & 0.92 & $0.83-0.96$ & 0.99 & $0.99-1.00$ & 0.98 & $0.97-0.95$ \\
\hline 7 & 0.99 & $0.99-0.99$ & 0.92 & $0.83-0.96$ & 1.00 & $0.99-1.00$ & 0.99 & $0.99-1.00$ \\
\hline 8 & 0.99 & $0.99-1.00$ & 0.97 & $0.94-0.99$ & 1.00 & 1.00 & 0.99 & $0.97-0.99$ \\
\hline 9 & 1.00 & $1.00-1.00$ & 0.99 & $0.99-0.99$ & 1.00 & 1.00 & 1.00 & $0.99-1.00$ \\
\hline
\end{tabular}

ICC, intraclass correlation coefficient; $\mathrm{Cl}$, confidence interval; $\mathrm{FBSI}$, during free-breathing, using the stability index; FBeye, during free-breathing, without using the stability index; BHSI, during breath-holding, using the stability index; BHeye, during breath-holding, without using the stability index. 
24 SWE measurements (8.3\%). In the breath-holding group, the number of unreliable results was four out of 27 patients (14.8\%) and there were no unreliable results when the $\mathrm{SI}$ was used.

An ICC value of $0.87(95 \% \mathrm{Cl}, 0.74$ to 0.94$)$ was found in the free-breathing group, and it increased to $0.99(95 \% \mathrm{Cl}, 0.97$ to 0.99$)$ when the SI was used. In the breath-holding group, the ICC value was $0.95(95 \% \mathrm{Cl}, 0.90$ to 0.98$)$, and it increased to $0.99(95 \% \mathrm{Cl}$, 0.99 to 0.99 ) when the $\mathrm{SI}$ was used.

The ICC values and $95 \%$ Cls calculated from comparing the mean of eight subsets of two to nine consecutive measurements and the reference values (median liver stiffness of the 10 measurements in each dataset) are summarized in Table 3. An ICC value over 0.95 implies that the measurement error due to the sampling number was less than $5 \%$, which is within the accepted error range [11]. In the free-breathing group, the first eight and nine repeated measurements showed ICC values over 0.95 compared with the reference values. With the use of $\mathrm{SI}$, the first five measurements and above had ICC values over 0.95 in comparison with the reference values. This means that the minimal number of repeated measurements with an acceptable error range decreased from eight to five repeated measurements when the SI was used. In the breathholding group, the first six repeated measurements compared with the reference values had ICC values over 0.95 when the SI was not used, whereas the first two repeated measurements demonstrated an ICC value of 0.99 . Using the SI reduced the minimal number of repeated measurements with an acceptable error range from six to two measurements in the breath-holding group.

\section{Discussion}

When measuring liver elasticity with US, issues related to the variability and reliability of elasticity measurements are quite important, especially in children who tend to be uncooperative and cannot hold their breath. Therefore, any technique that can decrease measurement variability is valuable. The $\mathrm{SI}$ is a newly introduced technique for reducing the number of unreliable results due to poor image acquisition and suboptimal Q-box positioning when SWE is performed to assess the degree of fibrosis within the liver. This tool, based on SWE coding conditions and temporal stability, automatically calculates the quality of each acquired image in order to obtain an SI value. Taking into consideration temporal, spatial, and noise information before calculating the IQR, the SI calculation helps users to rule out a poor-quality signal. When the $\mathrm{SI}$ is lower than $90 \%$, manufacturers recommend locating the Q-Box elsewhere in the 2-dimensional SWE box or performing the SWE acquisition again to reduce the number of poor SWE acquisitions and incorrect Q-Box locations.
Our study showed that variability in liver stiffness measurements could be reduced by using SI values. The standard deviation of SWE measurements and the coefficient of variation were reduced by applying SI values, which could be interpreted as a decreased spread of the variability of measurements relative to the mean, thereby providing more consistent and reliable measurements in both the free-breathing and breath-holding groups. Additionally, using SI not only decreased the number of unreliable results, but also increased intraobserver agreement in both the free-breathing and breathholding groups. Reliable measurements of liver stiffness are critical for determining and observing liver fibrosis and planning future treatment in clinical practice. Liver stiffness could be adequately measured with increased reliability and reduced inconsistency using the $\mathrm{SI}$, even when acquiring measurements during free breathing.

It is very important to reduce the scan time as much as possible in children, because it is particularly difficult to obtain adequate cooperation from patients to hold their breath or remain in the same position while measuring liver stiffness. Therefore, several previous studies have tried to identify the minimum number of acquisitions needed to obtain the optimal elasticity value in both adults and children $[16,18-20]$. In addition to these efforts, we speculate that we could further reduce the optimal minimal number of repeated measurements of SWE in both the free-breathing and breath-holding groups by using SI. Considering a sampling error range of less than $5 \%$ as acceptable, our results showed that the minimal number of repeated measurements decreased from eight to five repeated measurements by using the $\mathrm{SI}$ in the free-breathing group, and from six to two repeated measurements in the breath-holding group. Our hope is to reduce the number of repeated SWE measurements by utilizing the $\mathrm{SI}$, and eventually, reducing the scan time in children. In particular, referring to the $\mathrm{SI}$, we can expect not only decreased variability of liver stiffness measurements, but also a decreased scan time for adequate liver stiffness measurements, which can be very helpful when performing SWE in children. Since the optimal number of liver stiffness measurements can vary according to children's age [18], further investigation is needed to establish the role of the SI in reducing the number of liver stiffness measurements needed for an adequate assessment of liver stiffness in children.

However, our study also showed that acquiring SI values over $90 \%$ was not always possible because of significant temporal and spatial instability observed in some cases. In particular, the failure rate increased to $17 \%$ during free breathing due to respiratory movement. We suggest that vigorous breathing movements and underlying liver parenchymal heterogeneity may have contributed to this phenomenon. Therefore, we think that further investigation should be conducted into the cause of failure to obtain a sufficient SI value and that technical improvements should be made in order 
for this technique to be utilized consistently. With regard to the effect of breathing status on the liver SWE value, the study by Pellot-Barakat et al. [21] showed that the mean SWE value was higher in the measurements obtained during breath-holding than in those obtained during free breathing. They hypothesized that the hemodynamic changes during the different stages of breathing may lead to variability in SWE in the same patient [21]. Our study revealed a similar tendency for a higher stiffness value during free breathing, but without statistical significance.

There are several limitations in our study. First, our study cohort was rather small, and older children and young adults (with mean age of 16.1 years) were recruited, because our intention was to evaluate the effect of breathing status on utilization of SI. Even though we believe that our results could be applicable to young children who cannot hold their breath, a further study would be helpful to validate our results in young children. Second, patients with a variety of underlying diseases and a wide range of liver SWE values were included in our study. However, our study cohort reflects the normal patient distribution in routine clinical practice. Third, a single operator performed the SWE examinations. Therefore, interoperator variability could not be evaluated in our study. Lastly, due to limited patient cooperation in pediatric patients, it might be difficult to obtain images and precise measurements of SWE values during the free-breathing and breath-holding phases with strict control of respiration. However, we performed a large number of repeated measurements to acquire mean SWE values to overcome this shortcoming.

In conclusion, utilizing the $\mathrm{SI}$ in the measurement of liver SWE in children and young adults reduced measurement variability in both free-breathing and breath-holding conditions, and it may reduce the scan time by decreasing the number of repeated measurements in children and young adults.

ORCID: Eun Kyoung Hong: http://orcid.org/0000-0002-5440-0451; Young Hun Choi: http://orcid.org/0000-0002-1842-9062; Jung-Eun Cheon: http://orcid.org/00000003-1479-2064; Woo Sun Kim: http://orcid.org/0000-0003-2184-1311; In-One Kim: http://orcid.org/0000-0001-5800-3536; Sun Young Kang: http://orcid.org/00000003-1204-6287

\section{Conflict of Interest}

No potential conflict of interest relevant to this article was reported.

\section{References}

1. Han KH, Yoon KT. New diagnostic method for liver fibrosis and cirrhosis. Intervirology 2008;51 Suppl 1:11-16.

2. Tsochatzis EA, Gurusamy KS, Ntaoula S, Cholongitas E, Davidson
BR, Burroughs AK. Elastography for the diagnosis of severity of fibrosis in chronic liver disease: a meta-analysis of diagnostic accuracy. J Hepatol 2011;54:650-659.

3. Van Thiel DH, Gavaler JS, Wright H, Tzakis A. Liver biopsy: its safety and complications as seen at a liver transplant center. Transplantation 1993;55:1087-1090.

4. Xanthakos SA, Podberesky DJ, Serai SD, Miles L, King EC, Balistreri $W F$, et al. Use of magnetic resonance elastography to assess hepatic fibrosis in children with chronic liver disease. J Pediatr 2014;164:186-188.

5. Pariente D, Franchi-Abella S. Paediatric chronic liver diseases: how to investigate and follow up? Role of imaging in the diagnosis of fibrosis. Pediatr Radiol 2010;40:906-919.

6. Nobili V, Vizzutti F, Arena U, Abraldes JG, Marra F, Pietrobattista $A$, et al. Accuracy and reproducibility of transient elastography for the diagnosis of fibrosis in pediatric nonalcoholic steatohepatitis. Hepatology 2008;48:442-448.

7. Jeong WK, Lim HK, Lee HK, Jo JM, Kim Y. Principles and clinical application of ultrasound elastography for diffuse liver disease. Ultrasonography 2014;33:149-160.

8. Bavu E, Gennisson JL, Couade M, Bercoff J, Mallet V, Fink M, et al. Noninvasive in vivo liver fibrosis evaluation using supersonic shear imaging: a clinical study on 113 hepatitis $C$ virus patients. Ultrasound Med Biol 2011;37:1361-1373.

9. Dillman JR, Heider A, Bilhartz JL, Smith EA, Keshavarzi N, Rubin JM, et al. Ultrasound shear wave speed measurements correlate with liver fibrosis in children. Pediatr Radiol 2015;45:1480-1488.

10. Hudson JM, Milot L, Parry C, Williams R, Burns PN. Inter- and intraoperator reliability and repeatability of shear wave elastography in the liver: a study in healthy volunteers. Ultrasound Med Biol 2013;39:950-955.

11. Yoon JH, Lee JM, Han JK, Choi BI. Shear wave elastography for liver stiffness measurement in clinical sonographic examinations: evaluation of intraobserver reproducibility, technical failure, and unreliable stiffness measurements. J Ultrasound Med 2014;33:437447.

12. Woo H, Lee JY, Yoon JH, Kim W, Cho B, Choi BI. Comparison of the reliability of acoustic radiation force impulse imaging and supersonic shear imaging in measurement of liver stiffness. Radiology 2015;277:881-886.

13. Huang Y, Liu GJ, Liao B, Huang GL, Liang JY, Zhou LY, et al. Impact factors and the optimal parameter of acoustic structure quantification in the assessment of liver fibrosis. Ultrasound Med Biol 2015;41:2360-2367.

14. Sporea I, Gradinaru-Tascau O, Bota S, Popescu A, Sirli R, Jurchis $A$, et al. How many measurements are needed for liver stiffness assessment by 2D-shear wave elastography (2D-SWE) and which value should be used: the mean or median? Med Ultrason 2013;15:268-272. 
15. Everitt BS. The Cambridge dictionary of statistics. Cambridge: Cambridge University Press, 1998.

16. Ferraioli G, Tinelli C, Zicchetti M, Above E, Poma G, Di Gregorio M, et al. Reproducibility of real-time shear wave elastography in the evaluation of liver elasticity. Eur I Radiol 2012;81:3102-3106.

17. Busing KA, Kilian AK, Schaible T, Debus A, Weiss C, Neff KW. Reliability and validity of MR image lung volume measurement in fetuses with congenital diaphragmatic hernia and in vitro lung models. Radiology 2008;246:553-561.

18. Shin HJ, Kim MJ, Kim HY, Roh YH, Lee MJ. Optimal acquisition number for hepatic shear wave velocity measurements in children.
PLoS One 2016;11:e0168758.

19. Huang ZP, Zhang XL, Zeng J, Zheng J, Wang P, Zheng RQ. Study of detection times for liver stiffness evaluation by shear wave elastography. World J Gastroenterol 2014;20:9578-9584.

20. Choi SH, Jeong WK, Kim Y, Lim S, Kwon JW, Kim TY, et al. How many times should we repeat measuring liver stiffness using shear wave elastography?: 5-repetition versus 10-repetition protocols. Ultrasonics 2016;72:158-164.

21. Pellot-Barakat C, Chami L, Correas JM, Lefort M, Lucidarme O. Does motion affect liver stiffness estimates in shear wave elastography? Phantom and clinical study. Eur J Radiol 2016;85:1645-1650. 